\title{
ASSESSING CONSTRUCTION LABOURS' SAFETY LEVEL: A FUZZY MCDM APPROACH
}

\author{
Saeed Reza MOHANDES ${ }^{1}$, Haleh SADEGHI ${ }^{2}$, Amir MAHDIYAR $^{3}$, Serdar DURDYEV ${ }^{*}$, \\ Audrius BANAITIS ${ }^{5}$, Khairulzan YAHYA ${ }^{3}$, Syuhaida ISMAIL ${ }^{4}$ \\ ${ }^{1}$ Department of Civil and Environmental Engineering, The Hong Kong University of Science and Technology, \\ Clear Water Bay, Kowloon, Hong Kong \\ ${ }^{2}$ Department of Architectural Engineering, Islamic Azad University, Science and Research Branch, Shiraz, Iran \\ ${ }^{3}$ School of Civil Engineering, Faculty of Engineering, Universiti Teknologi Malaysia, 81310 Johor, Malaysia \\ ${ }^{4}$ Razak Faculty of Technology and Informatics, Universiti Teknologi Malaysia, Jalan Sultan Yahya Petra, \\ 54100 Kuala Lumpur, Malaysia \\ ${ }^{5}$ Department of Construction Management and Real Estate, Vilnius Gediminas Technical University, \\ 10223 Vilnius, Lithuania
}

Received 24 April 2019; accepted 03 September 2019

\begin{abstract}
Risk decision matrix has widely been favoured by the researchers in the area of construction safety risk assessment. Although it provides the construction safety professionals with the final illustration of the risks magnitude, it suffers from major shortcomings, including inability to considering the importance of probability and severity, impaired analysis resulting from the use of raw numbers for ratings, and the limited range of classifications for assessing the risks. All these shortages give an impaired insight to the concerned parties, deteriorating the involved workers' safety. As such, this paper aims to develop a novel Risk Assessment Model (RAM) through the integration of the Fuzzy Best Worst Method (FBWM) with the Interval-Valued Fuzzy Technique for Order of Preference by Similarity to Ideal Solution (IVFTOPSIS). Based on the application of RAM to a real-life case study, it was observed that the developed RAM contributes to the body of construction safety risk assessment in five unique ways: (1) computing the importance of the two risk parameters (i.e. probability and severity) using fuzzy-reference-based comparisons, (2) obviating the needs for having statistical data, (3) prioritizing the identified risks using the combination of interval-valued triangular fuzzy numbers with TOPSIS, (4) providing the safety analysts with wider ranges of classifications for conducting risk assessment, and (5) providing the safety professionals with appropriate evaluation strategies for controlling the analysed risks. The developed model in the study can be applied to any projects, giving a conclusive plan to the concerned safety professionals for adopting the further prudent mitigation measurements.
\end{abstract}

Keywords: safety risk assessment, construction safety, interval-valued fuzzy number, multi-criteria decision-making method, fuzzy best-worst method, fuzzy TOPSIS, interval-valued fuzzy TOPSIS.

\section{Introduction}

Due to the frequent observation of lethal and non-lethal occupational injuries occurred in the construction sector, it has always been regarded as a notorious sector (Tam et al., 2004). Needless to say, the safety and health of the involved workers not only can be deteriorated, but also it significantly impairs the associated economics (Hinze et al., 2006). To make it more explicit, it is reported that the construction industry accounts for at least 60,000 of the fatal accidents occurred in the workplaces. Notably, this figure for the industrialized countries varies from $25 \%$ to $50 \%$ of the total fatal accidents occurred in workplaces (International Labour Organization, 2014). In light of this, conducting research on the area of safety risk assessment seems an undeniable fact, in order to reduce the occupational injuries and fatalities of the involved workers (Pinto et al., 2011).

In order to improve the construction workers' safety, some researchers have focused on the area of risk assessment. Hallowell (2008) reported a safety risk assessment approach for assessing safety level of different construc-

*Corresponding author. E-mail: sdurdyev@gmail.com 
tion trades. In another study, Aneziris et al. (2010) proposed a model using the assessment of occupational risks, with an emphasis on the quantification of a tunnel construction. In the same year, Mitropoulos and Namboodiri (2010) presented the findings from the initial implementation of task demand assessment and demonstrates its feasibility and applicability on two different operations: a roofing activity and a concrete paving operation. Based on the utilization of Analytical Hierarchy Process (AHP), Aminbakhsh et al. (2013) developed a safety model for assessing the risks that might happen on the construction sites. In their study, Papazoglou et al. (2017) proposed a model that quantifies relationship between the site conditions and worker's behaviour towards an accident.

As cited by some researchers (Faber \& Stewart, 2003; Nilsen \& Aven, 2003), due to the uncertainty and incompletion that are inherent in probabilistic-based or statistical-based assessment approaches, Fuzzy Inference System (FIS) has widely been taken into consideration as a beneficial feature to cope with the afore-mentioned drawbacks (Amiri et al., 2017). In order to support construction companies with their responsibilities to reduce occupational safety risks, Pinto (2014) presented the newly-developed fuzzy assessment model. Due to the incompletion of the data associated with the reportable accidents, Debnath et al. (2016) fostered a safety risk assessment approach, considering the accidents happened to different parts of body, the associated safety measures, and the costs associated with the provision of those measurements.

To assess the safety risks that threaten the lives of different working trades involved in construction activities, Amiri et al. (2017) developed a fuzzy rule-based system. In another study, Gunduz et al. (2016) developed a fuzzy structural equation model for development of a safety performance index in construction sites. Mohandes and Zhang (2019) developed a comprehensive framework for making improvement in the assessment of risks in the construction sector, based on the exploitation of traditional risk matrix concept.

With respect to the above-mentioned literature on the developed risk assessment models focused on the construction workers, the current study aims to tackle the following research questions:

1) What is the significance level of the two essential risk parameters (i.e. probability and severity) while assessing construction safety risk?

2) How can calculate the magnitude of the risks posing danger to the construction workers with respect to the consideration of the importance of two essential risk parameters?

3) How can provide the safety analysts with wider ranges of classifications for conducting risk assessment?

4) What should the evaluation strategy be for the analysed risks? Do they need to be controlled with the same level of consideration? Which one should be given more consideration?
The unaddressed questions stated above leads to adopting flawed deeds by the concerned safety professionals, which in turn, this deteriorates the safety and health of the involved construction workers. As a result, this study aims to develop a RAM for improving the construction workers' safety by the integration of FBWM and IVFTOPSIS. The developed RAM in the study can be applied to any construction projects, providing a prudent plan for the responsible safety professionals to tackle the identified risks based upon their calculated final rankings along with the provision of apposite evaluation strategies.

The paper starts with various steps of the developed RAM, including a brief exposition on the fuzzy systems in Section 1. Section 2 provides the results of the application of the developed model to a case study. Section 3 discusses the findings obtained from the application of the developed model, while conclusion together with future research to be conducted and limitation of the research are provided at the end of paper.

\section{Research methodology}

This study develops a novel hybrid RAM for assessing safety risks of the workers involved in the construction activities. Figure 1 illustrates the fostered algorithm containing three steps, namely the exploitation of FBWM to obtain the relative importance weights of the two essential risk parameters (i.e. severity and probability), the exploitation of the IVFTOPSIS to attain the final ranking of the identified risks, and the proposal of appropriate evaluation strategies with respect to the magnitude of the analysed risks. The major reason for the exploitation of the combined fuzzy approach in the study lies in two facts: the need for obtaining the real data (i.e. observed data associated with uncertainty and incompletion) is obviated, and more importantly, obtaining a precise final ranking of the risks based on the importance weights of two risk parameters concurrently through overcoming the human's subjectivity.

\subsection{A brief introduction to fuzzy inference system}

Prior to explaining the research framework developed in the study, a brief exposition on FIS together with its basic operations has been provided. Needless to say, FIS is subsumed under the umbrella of a broader category named as artificial intelligence (Mohandes et al., 2019). Being introduced by Zadeh (1965), FIS is a mathematical theory aims at modelling the imprecision or vagueness of human cognitive processes. The point of fuzzy set theory, which was adopted in this study, is that the degree of membership for an element is within a certain interval that is usually $[0,1]$ (Liou et al., 2008), where zero indicates that the element does not belong to the set. On the other hand, the membership value of one indicates that the element completely belongs to the set. Any value within the interval indicates that the element has a certain degree of membership or in other words, it has a partial belonging to the fuzzy set 


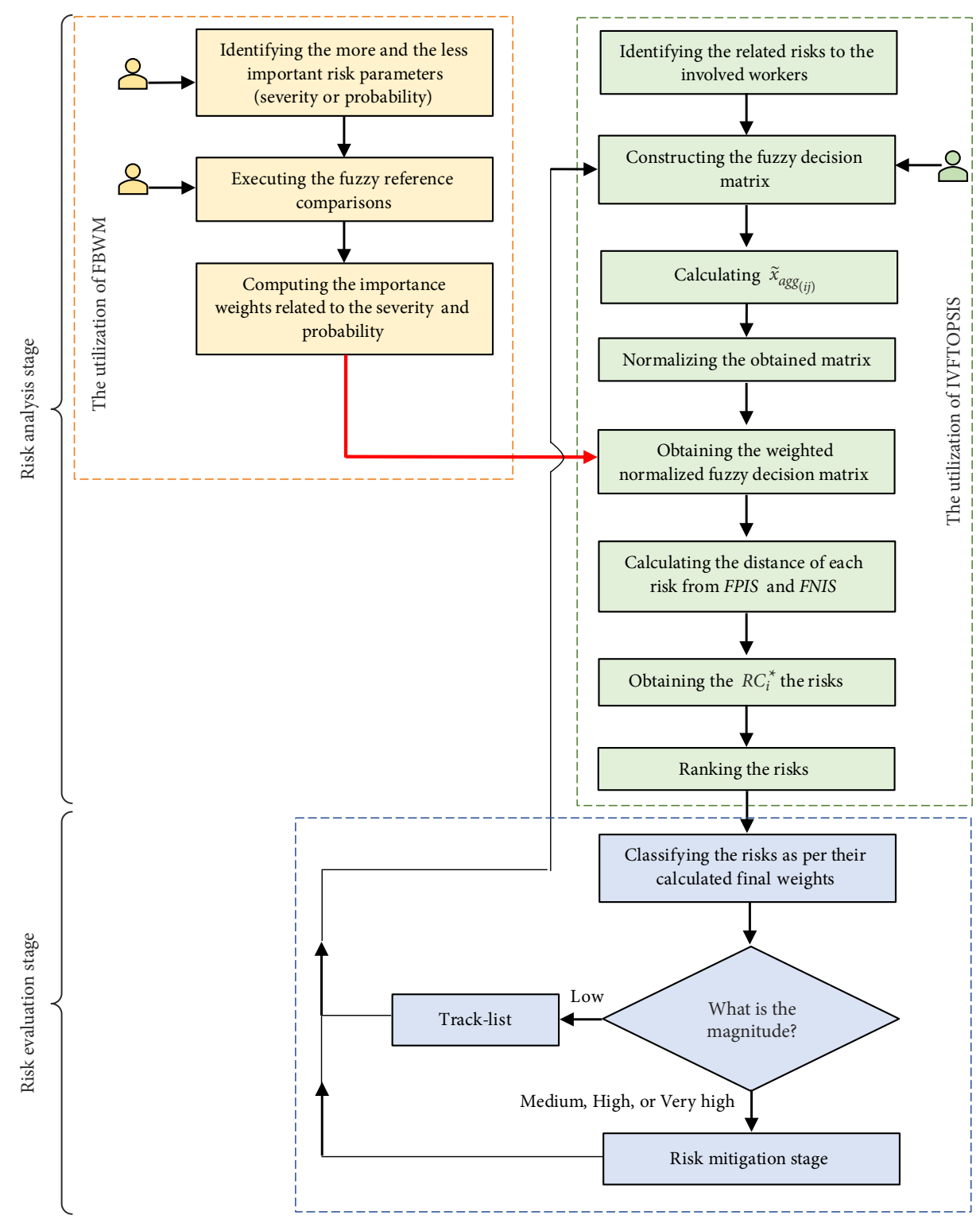

Figure 1. The developed RAM framework

(Armaghani et al., 2015; Bejarbaneh et al., 2018). A tilde will be placed above a symbol if the symbol represents a fuzzy set (e.g., $\tilde{A})$. A TFN is denoted simply as $(l|m, m| u)$ or $(l, m, u)$. Each TFN has linear representations on its left and right side such that its membership function can be defined as (Mikaeil et al., 2013):

$$
\mu(x \mid \tilde{A})= \begin{cases}0, & x\langle l \text { or } x\rangle u, \\ \frac{x-l}{m-l}, & l \leq x \leq m, \\ \frac{u-x}{u-m}, & m \leq x \leq u,\end{cases}
$$

where $l, m, u$ denote the lower bounds, the most likely value, and the upper bounds of the fuzzy number $\tilde{A}$. Figure 2 shows a TFN, including the lower bound, the most likely value, and the upper bound. The operational laws of TFNs $\tilde{A}_{1}$ and $\tilde{A}_{2}$ are as follows (Sun, 2010).
Addition of the two fuzzy numbers:

$\tilde{A}_{1} \tilde{A}_{2}=\left(l_{1}, m_{1}, u_{1}\right)\left(l_{2}, m_{2}, u_{2}\right)=\left(l_{1}+l_{2}, m_{1}+m_{2}, u_{1}+u_{2}\right)$.

Multiplication of the two fuzzy numbers:

$\tilde{A}_{1} \tilde{A}_{2}=\left(l_{1}, m_{1}, u_{1}\right)\left(l_{2}, m_{2}, u_{2}\right)=\left(l_{1}{ }_{2}, m_{1}{ }^{\star} m_{2}, u_{1}{ }^{\star} u_{2}\right)$.

Subtraction of the two fuzzy numbers:

$\tilde{A}_{1}-\tilde{A}_{2}=\left(l_{1}, m_{1}, u_{1}\right)-\left(l_{2}, m_{2}, u_{2}\right)=\left(l_{1}-u_{2}, m_{1}-m_{2}, u_{1}-l_{2}\right)$.

Division of the two fuzzy numbers:

$\tilde{A}_{1} \tilde{A}_{2}=\left(l_{1}, m_{1}, u_{1}\right)\left(l_{2}, m_{2}, u_{2}\right)=\left(l_{1} / u_{2}, m_{1} / m_{2}, u_{1} / l_{2}\right)$. 




Figure 2. TFNs representation

The graded mean integration representation of TFN $\tilde{A}$ (GMIR), i.e. the indicative of triangular fuzzy numbers ranking, can be calculated as below (Guo \& Zhao, 2017):

$$
R(\tilde{A})=\frac{l+4 m+u}{6} .
$$

It is noteworthy to point out that the interval-valued fuzzy numbers are special forms of generalized fuzzy numbers. Similar to generalized fuzzy numbers, these numbers can find a trapezoidal shape. For the sake of brevity, the readers could refer to Mondal (2016) for the operation laws and representations of interval-valued triangular fuzzy numbers.

\subsection{Background to risk assessment step}

Risk assessment step includes the identification of the critical risks along with the assessment of the identified risks in terms of their calculated magnitudes (Djapan et al., 2018; Hatami-Marbini et al., 2013; Zhao et al., 2016). Based on the concept of common risk matrix, the likelihood and intensity of each risk needs to be calculated, and subsequently they need to be multiplied by each other to give us the final magnitude (Mohandes \& Zhang, 2019). Meanwhile, it goes without saying that the application of MCDM methods can bring about fruitful results in the area of construction risk assessment, due to the fact that the assessment stage deals with different criteria, parameters, and alternatives with respect to each other (Tzeng \& Huang, 2011). To show the alignment of the application of MCDM methods to the construction risk assessment, the readers are referred to the following research used different techniques, such as the application of AHP (Zayed et al., 2008), the application of TOPSIS (Wang et al., 2008), and the application of ANP (Lu et al., 2008).

\subsection{FBWM}

The FBWM method, which is an integration of FIS into BWM (Guo \& Zhao, 2017), was adopted to compute the weights of criteria. As can be seen from Figure 1, the FBWM is exploited in the current research to obtain the importance of the probability and severity weights that will be used as the inputs into the IVFTOPSIS for obtain- ing the final orders of the identified risks endangering the involved workers. Due to the fact that FBWM utilizes reference-based comparison (Guo \& Zhao, 2017), which is in contrast to other MCDM methods (AHP, FAHP, ANP and FANP), a smaller number of pair-wise comparisons is sufficient to make an accurate ranking produced by the IVFTOPSIS. It is worthwhile to assert here that although the original BWM is based on the precise mathematical formulations, it is tangled with ambiguity as stated by Mohandes and Zhang (2019) due to the fact that raw numbers (i.e. in the range of 1 to 9) are used in the BWM, which increase the vagueness of the produced results. As such, the current study used FBWM, in which the mentioned ambiguity has overcome through the exploitation of FIS. The detailed steps involved in the execution of the FBWM in obtaining the importance weights pertaining to the two essential risk parameters, namely probability and severity, are as below.

Step 1. Defining the more and the less important parameters. As the initial step, the decision maker needs to define whether the probability or severity is of higher importance in the area of construction safety risk assessment. The more and less important parameters are named as $B$ and $W$, respectively henceforth.

Step 2. Executing the fuzzy reference comparisons. Once $B$ has been identified, the decision maker has to define the extent to which $B$ is more important than $W$ using the linguistic variables listed in Table 1 , contributing to the Fuzzy Best-to-Worst linguistic scale (known as $a_{B W}$ ).

Table 1. Linguistic variables for comparing the importance of two risk parameters

\begin{tabular}{|l|c|}
\hline \multicolumn{1}{|c|}{ Linguistic scales } & Membership function \\
\hline They have the same importance $(E)$ & $(1,1,1)$ \\
\hline $\begin{array}{l}\text { One parameter is weakly more } \\
\text { important than the other one }(W)\end{array}$ & $(2 / 3,1,3 / 2)$ \\
\hline $\begin{array}{l}\text { One parameter is moderately more } \\
\text { important than the other one }(M)\end{array}$ & $(3 / 2,2,5 / 2)$ \\
\hline $\begin{array}{l}\text { One parameter is strongly more } \\
\text { important than the other one }(S)\end{array}$ & $(5 / 2,3,7 / 2)$ \\
\hline $\begin{array}{l}\text { One parameter is extremely more } \\
\text { important than the other one }(E X)\end{array}$ & $(7 / 2,4,9 / 2)$ \\
\hline
\end{tabular}

Step 3. Finding the optimal weights $\left(\tilde{w}_{B^{*}}\right.$ and $\left.\tilde{w}_{W^{*}}\right)$.

In order to obtain the optimal weights related to the two risk parameters, the maximum absolute difference $\left|\frac{w_{B}}{w_{W}}-a_{B W}\right|$ needs to be minimized. Notably, $w_{B}$ and $w_{W}$ are both TFNs, and accordingly the fuzzy weights of either of the mentioned two parameters are represented as TFN $\tilde{w}_{j}=\left(l_{j}^{w}, u_{j}^{w}, m_{j}^{w}\right)$. For computing the relative weights, an optimization model could be built as shown below.

$$
\min \max _{j}\left\{\left|\frac{\tilde{w}_{B}}{\tilde{w}_{W}}-\tilde{a}_{B W}\right|\right\}
$$




$$
\text { s.t. }\left\{\begin{array}{l}
\sum_{j=1}^{2} R\left(\tilde{w}_{j}\right)=1 \\
l_{j}^{w} \leq m_{j}^{w} \leq u_{j}^{w}, \\
l_{j}^{w} \geq 0 \\
j=1,2
\end{array}\right.
$$

where $\tilde{w}_{B}=\left(l_{B}^{w}, m_{B}^{w}, u_{B}^{w}\right), \tilde{w}_{W}=\left(l_{W}^{w}, m_{W}^{w}, u_{W}^{w}\right), \tilde{w}_{j}=\tilde{w}_{B}$ and $\tilde{w}_{W}$, and $\tilde{a}_{B W}=\left(l_{B W}, m_{B W}, u_{B W}\right)$.

The following non-linear constrained optimization model is the transference of the model mentioned above:

$$
\begin{aligned}
& \min \tilde{\xi} \\
& \text { s.t. }\left\{\begin{array}{l}
\left|\frac{\tilde{w}_{B}}{\tilde{w}_{W}}-\tilde{a}_{B W}\right| \leq \tilde{\xi} \\
\sum_{j=1}^{n} R\left(\tilde{w}_{j}\right)=1 \\
l_{j}^{w} \leq m_{j}^{w} \leq u_{j}^{w} \\
l_{j}^{w} \geq 0 \\
j=1,2
\end{array},\right.
\end{aligned}
$$

where $\tilde{\xi}=\left(l \tilde{\xi}, m^{\tilde{\xi}}, u^{\tilde{\xi}}\right)$.

Considering $\tilde{l}_{\tilde{\xi}} \leq m^{\tilde{\xi}} \leq u^{\tilde{\xi}}$, it is supposed that $\widetilde{\xi^{*}}=$ $\left(k^{*}, k^{*}, k^{*}\right), k^{*} \leq l^{\tilde{\xi}}$, then Eq. (8) can be transferred as

$$
\begin{aligned}
& \min \widetilde{\xi}^{*} \\
& \text { s.t. }\left\{\begin{array}{l}
\left|\frac{\left(l_{B}^{W}, m_{B}^{W}, u_{B}^{W}\right)}{\left(l_{W}^{W}, m_{W}^{W}, u_{W}^{W}\right)}-\left(l_{B W}, m_{B W}, u_{B W}\right)\right| \leq\left(k^{*}, k^{*}, k^{*}\right) \\
\sum_{j=1}^{n} R\left(\tilde{w}_{j}\right)=1 \\
l_{j}^{w} \leq m_{j}^{w} \leq u_{j}^{w} \\
l_{j}^{w} \geq 0 \\
j=1,2
\end{array}\right.
\end{aligned}
$$

Considering the operational laws of TFNs, we can achieve the following model:

$\min k^{*}$

$$
\text { s.t. }\left\{\begin{array}{l}
\left|\frac{\left(l_{B}^{W}\right)}{\left(u_{W}^{W}\right)}-\left(l_{B W}\right)\right| \leq\left(k^{*}\right) \\
\left|\frac{\left(m_{B}^{W}\right)}{\left(m_{W}^{W}\right)}-\left(m_{B W}\right)\right| \leq\left(k^{*}\right) \\
\mid \frac{\left(u_{B}^{W}\right)}{\left(l_{W}^{W}\right)}-\left(u_{B W}\right) \\
n
\end{array} \mid \leq\left(k^{*}\right),\right.
$$

For solving the constrained optimization model built above, the optimal solution to $k^{*}$ should be reached as follows.

$$
\begin{aligned}
& \min k^{*} \\
& \text { s.t. }\left\{\begin{array}{l}
\mid\left(\begin{array}{l}
\left(l_{B}^{W}\right)-\left(l_{B W}\right) *\left(u_{W}^{W}\right) \mid \leq\left(k^{*}\right) *\left(u_{W}^{W}\right) \\
\left(m_{B}^{W}\right)-\left(m_{B W}\right) *\left(m_{W}^{W}\right) \mid \leq\left(k^{*}\right) *\left(m_{W}^{W}\right) \\
\left(u_{B}^{W}\right)-\left(u_{B W}\right) *\left(l_{W}^{W}\right) \mid \leq\left(k^{*}\right) *\left(l_{W}^{W}\right) \\
\sum_{j=1}^{n} R\left(\tilde{w}_{j}\right)=1 \\
l_{j}^{w} \leq m_{j}^{w} \leq u_{j}^{w} \\
l_{j}^{w} \geq 0 \\
j=1,2
\end{array}\right.
\end{array}\right.
\end{aligned}
$$

Solving the problem, the relative optimal weights to the importance of the probability and severity, namely $\tilde{w}_{B^{*}}$ and $\tilde{w}_{W^{*}}$ could be obtained. Obviously, since there is only one comparison that needs to be made, the model is of utmost consistency (i.e. Consistency Index equals 1).

Step 4. Defuzzification. Once the $\tilde{w}_{B^{*}}$ and $\tilde{w}_{W^{*}}$ have been obtained, they need to be defuzzified as below.

$$
\begin{aligned}
& w_{B^{*}}=\frac{l_{B^{*}}^{w}+4 m_{B^{*}}^{w}+u_{B^{*}}^{w}}{6} ; \\
& w_{W^{*}}=\frac{l_{W^{*}}^{w}+4 m_{W^{*}}^{w}+u_{W^{*}}^{w}}{6} .
\end{aligned}
$$

Step 5. Aggregating the computed weights. After the calculation of $w_{B^{*}}$ and $w_{W^{*}}$ by $\mathrm{K}$ experts, the computed weights of the probability and severity that were obtained from all the experts need to be aggregated as follows.

$$
W_{a g g_{j}}=\frac{1}{K} \times\left[W_{a_{1}}+W_{a_{2}}+\ldots+W_{a_{k}}\right], j=1,2,
$$

where $W_{a}$ indicates the calculated weights related to the either severity or probability of the Kth expert's appraisal. Notably, two aggregated weights for each risk need to be calculated; one for probability, and the other one for severity (i.e. because $j=1,2$ ).

\subsection{IVFTOPSIS}

The TOPSIS method - introduced by Hwang and Yoon (1981) - was used to make a decision on the best alternative as per the concepts of compromised solution. The compromised solution can be defined as choosing a solution with respect to the shortest distance from the ideal solution and the farthest distance from the negative ideal solution. To avoid subjectivity of human's judgement, who are using raw numbers, an extended TOPSIS with the application of fuzzy numbers was developed, as recommended by Celik et al. (2012) and Tzeng and Huang (2011).

This study exploits the IVFTOPSIS to systematically obtain a precise final ranking of the risks that were identified to be potentially influential on construction workers. Once the relative importance weights of the two risk pa- 
rameters have been determined using the FBWM in the previous step, the obtained weights need to be fed as the input to the IVFTOPSIS. This study adopts the IVFTOPSIS proposed by Ashtiani et al. (2009), as IVTFNs captures more uncertainty than the commonly used TFNs. The detailed steps of the exploitation of the IVFTOPSIS in obtaining the final orders of the defined risks posing danger to the construction workers are as follows.

Step 1. Constructing the fuzzy decision matrix to choose the suitable linguistic variables for the identified risks regarding the two risk parameters (see Table 2). Based on the experts' points of views, the fuzzy decision matrix that includes the two essential parameters of risk (i.e. probability and severity) and m number of risks could be formed as below:

$$
R D M=\left[\begin{array}{cc}
x_{11} & x_{12} \\
\vdots & \vdots \\
x_{m 1} & x_{m 2}
\end{array}\right] .
$$

It is noteworthy to mention that criteria and alternatives existing in the common TOPSIS method in this study are referred to as the risk parameters (i.e. probability and severity) and the different types of risks, respectively.

Step 2. Calculating the aggregated weights. Once the relative experienced decision makers' views have been obtained, the IVTFNs given to each risk with respect to the probability and severity need to be aggregated. To calculate the aggregated value for each risk, the following steps need to be followed.

Assuming that $\tilde{x}_{i j k}=\left[\left(l, l^{\prime}\right)_{i j k}, m_{i j k},\left(u, u^{\prime}\right)_{i j k}\right]$ is the fuzzy score of the risk $i$ with regard to the risk parameter $j$ that is given by the number $k$ expert, for $i=1,2, \ldots, m$, $j=1,2$, and $k=1,2, \ldots, n$. Then, the aggregated fuzzy weight of all the experts' appraisal can be calculated as follows:

$$
\tilde{x}_{a g g_{(i j)}}=\frac{1}{K} \times\left[\tilde{x}_{i j 1}+\tilde{x}_{i j 1}+\ldots+\tilde{x}_{i j k}\right] .
$$

Step 3. Obtaining the normalized fuzzy risk decision matrix (i.e. $\left[\tilde{r}_{i j}\right]_{n \times 2}$ ). Using the below-mentioned equa- tions, the aggregated matrix obtained in the previous step could be normalized:

$$
\begin{aligned}
& \tilde{r}_{i j}=\left[\left(\frac{l_{i j}}{u_{j}^{+}}, \frac{l_{i j}^{\prime}}{u_{j}^{+}}\right) ; \frac{m_{i j}}{u_{j}^{+}} ;\left(\frac{u_{i j}^{\prime}}{u_{j}^{+}}, \frac{u_{i j}}{u_{j}^{+}}\right)\right], i=1,2, \ldots, m, j \in \Omega_{b} ; \\
& \tilde{r}_{i j}=\left[\left(\frac{l_{j}^{-}}{l_{i j}^{\prime}}, \frac{l_{j}^{-}}{l_{i j}}\right) ; \frac{l_{j}^{-}}{m_{i j}} ;\left(\frac{l_{j}^{-}}{u_{i j}}, \frac{l_{j}^{-}}{u_{i j}^{\prime}}\right)\right], i=1,2, \ldots, m, j \in \Omega_{c},
\end{aligned}
$$

where $u_{j}^{+}=\max _{i} u_{i j}^{+}$and $l_{j}^{-}=\min _{i} l_{i j}^{\prime}$.

Step 4. Constructing the weighted normalized fuzzy decision matrix (i.e. $\left[\tilde{v}_{i j}\right]_{n \times 2}$ where $\tilde{v}_{i j}=W_{a g g_{j}} \times \tilde{r}_{i j}$ ). According to the operational laws of IVTFNs, we have the following:

$$
\begin{aligned}
& \tilde{v}_{i j}=\left[\left(\tilde{r}_{i j} \times w_{a g g_{j}}, \tilde{r}_{i j}^{\prime} \times w_{a g g_{j}}\right) ; \tilde{r}_{m_{i j}} \times w_{a g g_{j}} ;\right. \\
& \left.\left(\tilde{r}_{u_{i j}} \times w_{a g g_{j}}, \tilde{r}_{u_{i j}^{\prime}} \times w_{a g g_{j}}\right)\right]=\left[\left(a_{i j}, a_{i j}^{\prime}\right) ; b_{i j},\left(c_{i j}^{\prime}, c_{i j}\right)\right] .
\end{aligned}
$$

Step 5. Finding ideal solutions. Fuzzy Positive Ideal Solution (FPIS) and Fuzzy Negative Ideal Solution (FNIS) can be defined as below:

$$
\begin{aligned}
& F P I S=[(1,1) ; 1 ;(1,1)], \quad j \in \Omega_{b} ; \\
& F N I S=[(0,0) ; 0 ;(0,0)], \quad j \in \Omega_{c} .
\end{aligned}
$$

Step 6. Calculating the distance of each risk from FPIS and FNIS. At this stage, the distance of risks from the FPIS (known as $D_{i 1}^{+}$and $D_{i 2}^{+}$) need to be calculated as follows:

$$
\begin{aligned}
& D_{i 1}^{+}=\sum_{j=1}^{2} \sqrt{\frac{1}{3}\left[\left(a_{i j}-1\right)^{2}+\left(b_{i j}-1\right)^{2}+\left(c_{i j}-1\right)^{2}\right]} ; \\
& D_{i 2}^{+}=\sum_{j=1}^{2} \sqrt{\frac{1}{3}\left[\left(a_{i j}^{\prime}-1\right)^{2}+\left(b_{i j}-1\right)^{2}+\left(c_{i j}^{\prime}-1\right)^{2}\right]} .
\end{aligned}
$$

Table 2. Linguistic scales for the evaluation of risks with respect to the two risk parameters

\begin{tabular}{|l|l|l|l|c|}
\hline \multicolumn{1}{|c|}{ Variables for probability } & \multicolumn{1}{|c|}{ Description } & \multicolumn{1}{|c|}{ Variables for severity } & \multicolumn{1}{|c|}{ Description } & \multicolumn{1}{|c|}{ IVTFNs } \\
\hline $\begin{array}{l}\text { Extremely low probable } \\
\text { (ELP) }\end{array}$ & Virtually impossible & $\begin{array}{l}\text { Extremely low severe } \\
\text { (ELS) }\end{array}$ & First aid & {$[(0,0) ; 0 ;(1,1.5)]$} \\
\hline Very low probable (VLP) & Practically impossible & Very low severe $($ VLS) & Minor injury & {$[(0,0.5) ; 1 ;(2.5,3.5)]$} \\
\hline $\begin{array}{l}\text { Medium low probable } \\
\text { (MLP) }\end{array}$ & $\begin{array}{l}\text { Conceivable but very } \\
\text { unlikely }\end{array}$ & $\begin{array}{l}\text { Medium low severe } \\
\text { (MLS) }\end{array}$ & $\begin{array}{l}\text { Temporary } \\
\text { disability }\end{array}$ & {$[(0,1.5) ; 3 ;(4.5,5.5)]$} \\
\hline Medium probable (MP) & Only remotely possible & Medium severe (MS) & Serious injury & {$[(2.5,3.5) ; 5 ;(6.5,7.5)]$} \\
\hline $\begin{array}{l}\text { Medium highly probable } \\
\text { (MHP) }\end{array}$ & Unusual but possible & $\begin{array}{l}\text { Medium highly severe } \\
\text { (MHS) }\end{array}$ & $\begin{array}{l}\text { Permanent } \\
\text { disability }\end{array}$ & {$[(4.5,5.5) ; 7 ;(8,9.5)]$} \\
\hline $\begin{array}{l}\text { Very highly probable } \\
\text { (VHP) }\end{array}$ & Quite possible & Very highly severe (VHS) & fatality & {$[(5.5,7.5) ; 9 ;(9.5,10)]$} \\
\hline $\begin{array}{l}\text { Extremely highly probable } \\
\text { (EHP) }\end{array}$ & Might well be expected & $\begin{array}{l}\text { Extremely highly severe } \\
\text { (EHS) }\end{array}$ & Many fatalities & {$[(8.5,9.5) ; 10 ;(10,10)]$} \\
\hline
\end{tabular}


Similarly, the separation from the FNIS (known as $D_{i 1}^{-}$ and $D_{i 2}^{-}$) can be calculated as below:

$$
\begin{aligned}
& D_{i 1}^{-}=\sum_{j=1}^{2} \sqrt{\frac{1}{3}\left[\left(a_{i j}-0\right)^{2}+\left(b_{i j}-0\right)^{2}+\left(c_{i j}-0\right)^{2}\right]} ; \\
& D_{i 2}^{-}=\sum_{j=1}^{2} \sqrt{\frac{1}{3}\left[\left(a_{i j}^{\prime}-0\right)^{2}+\left(b_{i j}-0\right)^{2}+\left(c_{i j}^{\prime}-0\right)^{2}\right]} .
\end{aligned}
$$

Step 7. Obtaining the Relative Closeness. Using the below-mentioned equations, the final values of the respective risks (known as $R C_{i}^{*}$ ) can be obtained:

$$
\begin{aligned}
& R C_{1}=\frac{D_{i 2}^{-}}{D_{i 2}^{+}+D_{i 2}^{-}} ; \\
& R C_{2}=\frac{D_{i 1}^{-}}{D_{i 1}^{+}+D_{i 1}^{-}} ; \\
& R C_{i}^{*}=\frac{R C_{1}+R C_{2}}{2}
\end{aligned}
$$

Step 8. Obtaining the order of risks. In the final step, the risks need to be ranked based on their calculated relative closeness. The higher the relative closeness, the riskier the relative risk.

\subsection{Evaluation strategies}

As one may notice, a proper assessment stage should end in the provision of the decisions on how to evaluate the risks ranked in the previous step (Rausand, 2013). In the current study, it is aimed to come up with a straightforward evaluation plan for the safety experts regarding the ranked risks. To do so, the linear interpolation technique is taken into consideration. Firstly, due to the fact that the risks magnitude according to the common risk matrix are in the range of $[1,25]$, the final risks weights that were computed by the developed RAM needs to be set out within this range. In doing so, the minimum and the maximum calculated weights need to be assigned 1 and 25 , respectively, and accordingly the remaining weights need to be set out within the mentioned range using the linear interpolation technique. Following the calculation of the new weights related to all the risks, the following rules should be followed:

Rule A: If $1 \leq x \leq 4$, then the magnitude is low $\Rightarrow$ the respective risk could be accepted;

Rule B: If $4<x \leq 10$, then the magnitude is medium $\Rightarrow$ the respective risk should be mitigated;

Rule C: If $10<x \leq 16$, then the magnitude is high $\Rightarrow$ the respective risk should be mitigated;

Rule D: If $16<x \leq 25$, then the magnitude is very high $\Rightarrow$ the respective risk should be mitigated,

where $x$ is the newly-calculated weights of the respective risk using linear interpolation technique. It is notable to mention that the range of the numbers used within the range of common risk matrix are adopted from (Li et al., 2018). Once the suitable evaluation strategies for all the risks have been determined, the safety experts handling the project need to consider the following issues:

Consideration 1. New types of risks posing danger to the involved construction workers need to be identified, and accordingly they need to be assessed using the developed RAM. The timespan for determining the period to identify new risks is dependent on the project manager's sagacity. For instance, if the project manager decides on conducting the reassessment stage every six month, then the mentioned period for such identifications should be recurred every six months.

Consideration 2. The risks that are of low criticality (i.e. track-list risks) need to be reassessed periodically to be ensured regarding the fact that their magnitudes have not changed. However, the timespan for conducting the reassessment of the pertinent risks is less frequent as compared to the riskier risks (i.e. the risks to be mitigated).

Consideration 3. The risks needed to be mitigated (i.e. the risks which have medium, highly, or very highly magnitude) should be reassessed in order to be ensured regarding the fact that their magnitudes have decreased to the threshold level (i.e. low boundary). If the relative magnitude in the reassessment has not decreased to the satisfactory level, then the respective risks should go to the risk mitigation stage again. Otherwise, they need to be tracked periodically as discussed above. Notably, the reassessment of the critical risks to be mitigated needs to be conducted more frequently as against the low-criticality risks.

\section{Case study}

In this section, a real-life case study to show the practicability of the developed RAM is considered. The selected case study is a medium-sized company performing the services related to the maintenance of the lifts in Hong Kong. In view of anonymity reasons, the mentioned company's profile is not revealed. With this in mind, all the steps involved in the developed RAM are applied to the selected real-life case study henceforth.

\subsection{Risk identification stage}

As mentioned earlier, the first stage of risk assessment is the identification of the risks that may threaten the workers. As such, in order to identify all the critical risks related to the involved lift maintenance workers, a comprehensive literature review (Lee \& Lim, 2014; Mohandes \& Zhang, 2019; Zarikas et al., 2013) along with interviewing the relevant experienced experts were conducted. It was observed that the crucial risks endangering the involved lift maintenance workers are as follows: electrocution (S1), fire (S2), trapping (S3), fall from height (S4), struck (S5), back injury (S6), tendinitis (S7), neck injury (S8), dermatitis (S9), scald (S10), eye injury (S11). 


\subsection{Risk assessment stage}

Following the identification of the critical risks, they are needed to be assessed in terms of their criticalities. Hence, the step-by-step application of the developed RAM to the chosen case study is followed henceforth. It is notable to mention that in order to conduct the risk assessment stage, three experts of the mentioned company were selected, who had more than 15 years of working experience with respect to the safety of the workers performing the required maintenance services (see Table 3). According to the studies conducted by Yazdi (2018) and AbdelBasset et al. (2019), the limited numbers of experts used in MCDM-based methods (such AHP, ANP, or TOPSIS) should be three, and as such, the size of panel members for the current study is prudent enough. Additionally, Zhang et al. (2019) and Ren et al. (2017) asserted that when FIS has been integrated with MCDM methods, a size of panel consisting of three experts (decision makers) is enough to validate the applicability of any developed models, which results from a significant decrease in the involved decision makers' subjectivity.

Table 3. Experts' profile

\begin{tabular}{|c|l|l|}
\hline $\begin{array}{c}\text { Expert's } \\
\text { NO }\end{array}$ & \multicolumn{1}{|c|}{$\begin{array}{c}\text { Area of specialization } \\
\text { (degree) }\end{array}$} & Years of experience \\
\hline 1 & $\begin{array}{l}\text { Civil Engineering } \\
\text { (Undergrad) }\end{array}$ & Between 15 and 20 \\
\hline 2 & $\begin{array}{l}\text { Civil Engineering } \\
\text { (Undergrad) }\end{array}$ & Between 15 and 20 \\
\hline 3 & $\begin{array}{l}\text { Construction Management } \\
(\mathrm{PhD})\end{array}$ & More than 20 \\
\hline
\end{tabular}

\subsubsection{The exploitation of FBWM}

As shown in Figure 1, the FBWM is used in this study to assess the importance of the two essential risk parameters. The selected experienced experts were asked to make a fuzzy-reference-based comparison between the importance of probability and severity in the area of construction safety risk assessment. Table 4 shows the comparison made by the invited experts, including the computation of the relative weights and the aggregated weights. To aid the readers' understanding regarding the computation process of the FBWM exploitation, a sample questionnaire along with the relative computational processes are elaborated in the Appendixes A and B, respectively.

\subsubsection{The exploitation of IVFTOPSIS}

The relative experts were asked to determine the probability and severity of the identified risks using IVTFNs (Step 1), which is followed by the calculation of the aggregated weights of the identified risks (Step 2). Appendix C summarizes the comparisons made by one of the relative experts. Table 5 shows all the assessment made by the invited three experts for both probability and severity. Then, based on the steps 3 and 4 of the proposed IVFTOPSIS, the normalized fuzzy decision matrix together with the weighted normalized fuzzy decision matrix were calculated (see Table 6). Following this, in order to obtain the final ranking of the identified risks, steps 5-8 were conducted (see Table 7). Based on the obtained final ranking of the risks, the orders of the assessed risks regarding their criticality level can be set out as follows; $\mathrm{S} 3>\mathrm{S} 4>\mathrm{S} 6>\mathrm{S} 1>$ $\mathrm{S} 5>\mathrm{S} 2>\mathrm{S} 11>\mathrm{S} 8>\mathrm{S} 7>\mathrm{S} 9=\mathrm{S} 10$.

Table 4. Comparisons of the importance of two risk parameters

\begin{tabular}{|l|c|c|c|}
\hline \multicolumn{1}{|c|}{ Expert NO } & E1 & E2 & E3 \\
\hline (The chosen more important parameter, the extent of importance) & $(\mathrm{S}, \mathrm{M})$ & $(\mathrm{S}, \mathrm{W})$ & $(\mathrm{S}, \mathrm{W})$ \\
\hline Computed weights $[\mathrm{S}, \mathrm{P}]$ & {$[0.6641,0.3358]$} & {$[0.4994,0.5005]$} & {$[0.4994,0.5005]$} \\
\hline$W_{\text {agg }}[\mathrm{S}, \mathrm{P}]$ & & & {$[0.5543,0.4456]$} \\
\hline
\end{tabular}

Table 5. Evaluations of the risks with respect to the two risk parameters

\begin{tabular}{|c|c|c|c|c|c|c|c|c|}
\hline \multirow{2}{*}{ Risks } & \multicolumn{4}{|c|}{ Probability } & \multicolumn{4}{|c|}{ Severity } \\
\hline & $\mathrm{E} 1$ & E2 & E3 & $\tilde{x}_{a g g}$ & E1 & E2 & E3 & $\tilde{x}_{a g g}$ \\
\hline S1 & $\mathrm{VL}$ & $\mathrm{VL}$ & ML & {$[(0.00,0.83) ; 1.67 ;(3.17,4.17)]$} & $\mathrm{VH}$ & $\mathrm{EH}$ & $\mathrm{VH}$ & {$[(6.50,8.17,9.33,9.67,10.00)]$} \\
\hline S2 & EL & $\mathrm{EL}$ & $\mathrm{VL}$ & {$[(0.00,0.17,0.33,1.50,2.17)]$} & $\mathrm{VH}$ & $\mathrm{EH}$ & $\mathrm{VH}$ & {$[(6.50,8.17,9.33,9.67,10.00)]$} \\
\hline S3 & $\mathrm{MH}$ & $\mathrm{VH}$ & $\mathrm{VH}$ & {$[(5.17,6.83,8.33,9.00,9.83)]$} & $\mathrm{MH}$ & $\mathrm{MH}$ & $\mathrm{MH}$ & {$[(4.50,5.50,7.00,8.00,9.50)]$} \\
\hline S4 & ML & $\mathrm{M}$ & $M$ & {$[(1.67,2.83,4.33,5.83,6.83)]$} & $\mathrm{VH}$ & $\mathrm{EH}$ & $\mathrm{EH}$ & {$[(6.50,8.17,9.33,9.67,10.00)]$} \\
\hline S5 & $\mathrm{MH}$ & $\mathrm{VH}$ & $\mathrm{MH}$ & {$[(4.83,6.17,7.67,8.50,9.67)]$} & $\mathrm{ML}$ & $\mathrm{VL}$ & $\mathrm{M}$ & {$[(0.83,1.83,3.00,4.50,5.50)]$} \\
\hline S6 & $\mathrm{VH}$ & $\mathrm{VH}$ & $\mathrm{VH}$ & {$[(5.50,7.50,9.00,9.50,10.00)]$} & ML & $M$ & $\mathrm{M}$ & {$[(1.67,2.83,4.33,5.83,6.83)]$} \\
\hline S7 & ML & ML & EL & {$[(0.00,1.00,2.00,3.33,4.17)]$} & $\mathrm{VL}$ & ML & VL & {$[(0.00,0.83,1.67,3.17,4.17)]$} \\
\hline S8 & $\mathrm{MH}$ & $\mathrm{M}$ & $\mathrm{VL}$ & {$[(2.33,3.17,4.33,5.67,6.83)]$} & VL & ML & VL & {$[(0.00,0.83,1.67,3.17,4.17)]$} \\
\hline S9 & $\mathrm{VL}$ & $\mathrm{VL}$ & $\mathrm{VL}$ & {$[(0.00,0.50,1.00,2.50,3.50)]$} & $\mathrm{VL}$ & ML & $\mathrm{VL}$ & {$[(0.00,0.83,1.67,3.17,4.17)]$} \\
\hline S10 & $\mathrm{VL}$ & VL & $\mathrm{VL}$ & {$[(0.00,0.50,1.00,2.50,3.50)]$} & VL & ML & VL & {$[(0.00,0.83,1.67,3.17,4.17)]$} \\
\hline S11 & ML & ML & $\mathrm{VL}$ & {$[(0.00,1.17,2.33,3.83,4.83)]$} & $\mathrm{M}$ & $\mathrm{MH}$ & MH & {$[(3.83,4.83,6.33,7.50,8.83)]$} \\
\hline
\end{tabular}




\subsection{Risk evaluation stage}

As explained previously in the methodology section, after computing the final relative weights to the risks $\left(R C_{i}^{*}\right)$, they need to be set out in the range of 1 to 25 using lin- ear interpolation technique. Table 8 illustrates the newlycalculated weights related to all the risks along with the proposed evaluation strategies appertaining to them.

Table 6. Normalized fuzzy decision matrix and weighted normalized fuzzy decision matrix

\begin{tabular}{|c|c|c|c|c|}
\hline \multirow{2}{*}{ Risk } & \multicolumn{2}{|c|}{ Normalized fuzzy decision matrix } & \multicolumn{2}{c|}{ Weighted normalized fuzzy decision matrix } \\
\cline { 2 - 6 } & Probability & Severity & Probability & Severity \\
\hline S1 & {$[(0.00,0.08) ; 0.17 ;(0.32,0.42)]$} & {$[(0.65,0.82) ; 0.93 ;(0.97,1.00)]$} & {$[(0.00,0.04), 0.07 ;(0.14,0.19)]$} & {$[(0.36,0.45) ; 0.52 ;(0.54,0.55)]$} \\
\hline S2 & {$[(0.00,0.02) ; 0.03 ;(0.15,0.22)]$} & {$[(0.65,0.82) ; 0.93 ;(0.97,1.00)]$} & {$[(0.00,0.01) ; 0.01 ;(0.07,0.10)]$} & {$[(0.36,0.45) ; 0.52 ;(0.54,0.55)]$} \\
\hline S3 & {$[(0.52,0.68) ; 0.83 ;(0.90,0.98)]$} & {$[(0.45,0.55) ; 0.70 ;(0.80,0.95)]$} & {$[(0.23,0.30) ; 0.37 ;(0.40,0.44)]$} & {$[(0.25,0.30) ; 0.39 ;(0.44,0.53)]$} \\
\hline S4 & {$[(0.17,0.28) ; 0.43 ;(0.58,0.68)]$} & {$[(0.65,0.82) ; 0.93 ;(0.97,1.00)]$} & {$[(0.07,0.13) ; 0.19 ;(0.26,0.30)]$} & {$[(0.36,0.45) ; 0.52 ;(0.54,0.55)]$} \\
\hline S5 & {$[(0.48,0.62) ; 0.77 ;(0.85,0.97)]$} & {$[(0.08,0.18) ; 0.30 ;(0.45,0.55)]$} & {$[(0.22,0.27) ; 0.34 ;(0.38,0.43)]$} & {$[(0.05,0.10) ; 0.17 ;(0.25,0.30)]$} \\
\hline S6 & {$[(0.55,0.75) ; 0.90 ;(0.95,1.00)]$} & {$[(0.17,0.28) ; 0.43 ;(0.58,0.68)]$} & {$[(0.25,0.33) ; 0.40 ;(0.42,0.45)]$} & {$[(0.09,0.16) ; 0.24 ;(0.32,0.38)]$} \\
\hline S7 & {$[(0.00,0.10) ; 0.20 ;(0.33,0.42)]$} & {$[(0.00,0.08) ; 0.17 ;(0.32,0.42)]$} & {$[(0.00,0.04) ; 0.09 ;(0.15,0.19)]$} & {$[(0.00,0.05) ; 0.09 ;(0.18,0.23)]$} \\
\hline S8 & {$[(0.23,0.32) ; 0.43 ;(0.57,0.68)]$} & {$[(0.00,0.08) ; 0.17 ;(0.32,0.42)]$} & {$[(0.10,0.14) ; 0.19 ;(0.25,0.30)]$} & {$[(0.00,0.05) ; 0.09 ;(0.18,0.23)]$} \\
\hline S9 & {$[(0.00,0.05) ; 0.10 ;(0.25,0.35)]$} & {$[(0.00,0.08) ; 0.17 ;(0.32,0.42)]$} & {$[(0.00,0.02) ; 0.04 ;(0.11,0.16)]$} & {$[(0.00,0.05) ; 0.09 ;(0.18,0.23)]$} \\
\hline S10 & {$[(0.00,0.05) ; 0.10 ;(0.25,0.35)]$} & {$[(0.00,0.08) ; 0.17 ;(0.32,0.42)]$} & {$[(0.00,0.02) ; 0.04 ;(0.11,0.16)]$} & {$[(0.00,0.05) ; 0.09 ;(0.18,0.23)]$} \\
\hline S11 & {$[(0.00,0.12) ; 0.23 ;(0.38,0.48)]$} & {$[(0.38,0.48) ; 0.63 ;(0.75,0.88)]$} & {$[(0.00,0.05) ; 0.10 ;(0.17,0.22)]$} & {$[(0.21,0.27) ; 0.35 ;(0.42,0.49)]$} \\
\hline
\end{tabular}

Table 7. Final ranking of the risk factors

\begin{tabular}{|c|c|c|c|c|c|c|}
\hline Risk & {$\left[D_{i 1}^{+}, D_{i 2}^{+}\right]$} & {$\left[D_{i 2}^{-}, D_{i 1}^{-}\right]$} & $R C_{1}$ & $R C_{2}$ & $R C_{i}^{*}$ & Ranking \\
\hline S1 & {$[2.12,2.12]$} & {$[0.95,0.99]$} & 0.3095 & 0.3180 & 0.3137 \\
\hline S2 & {$[2.21,2.21]$} & {$[0.86,0.88]$} & 0.2793 & 0.2857 & 0.2825 & 6 \\
\hline S3 & {$[1.84,1.88]$} & {$[1.19,1.26]$} & 0.3879 & 0.4063 & 0.3971 & 1 \\
\hline S4 & {$[1.95,1.95]$} & {$[1.12,1.15]$} & 0.3656 & 0.3714 & 0.3685 & 2 \\
\hline S5 & {$[2.20,2.23]$} & {$[0.84,0.91]$} & 0.2732 & 0.2932 & 0.2832 & 5 \\
\hline S6 & {$[2.04,2.04]$} & {$[1.03,1.07]$} & 0.3345 & 0.3438 & 0.3391 & 3 \\
\hline S7 & {$[2.67,2.71]$} & {$[0.38,0.45]$} & 0.1217 & 0.1453 & 0.1335 & 9 \\
\hline S8 & {$[2.51,2.55]$} & {$[0.53,0.61]$} & 0.1716 & 0.1968 & 0.1842 & 8 \\
\hline S9 & {$[2.72,2.77]$} & {$[0.32,0.41]$} & 0.1039 & 0.1312 & 0.1176 & 10 \\
\hline S10 & {$[2.72,2.77]$} & {$[0.32,0.41]$} & 0.1039 & 0.1312 & 0.1176 & 10 \\
\hline S11 & {$[2.27,2.31]$} & {$[0.77,0.85]$} & 0.2496 & 0.2737 & 0.2617 & 7 \\
\hline
\end{tabular}

Table 8. Proposed evaluations with respect to the analysed risks using interpolation technique

\begin{tabular}{|c|c|c|c|c|}
\hline Risk & $R C_{i}^{*}$ & New weights & Criticality level & Evaluation strategy \\
\hline S1 & 0.3137 & 17.83 & Very high & Should go to mitigation stage \\
\hline S2 & 0.2825 & 15.15 & High & Should go to mitigation stage \\
\hline S3 & 0.3971 & 25 & Very high & Should go to mitigation stage \\
\hline S4 & 0.3685 & 22.54 & Very high & Should go to mitigation stage \\
\hline S5 & 0.2832 & 15.21 & High & Should go to mitigation stage \\
\hline S6 & 0.3391 & 20.01 & Very high & Should be tracked periodically \\
\hline S7 & 0.1335 & 2.36 & Low & Should go to mitigation stage \\
\hline S8 & 0.1842 & 6.71 & Medium & Should be tracked periodically \\
\hline S9 & 0.1176 & 1 & Low & Should be tracked periodically \\
\hline S10 & 0.1176 & 1 & Low & Should go to mitigation stage \\
\hline S11 & 0.2617 & 13.37 & High & \\
\hline
\end{tabular}




\subsection{Comparative analysis}

In this sub-section, the results produced by the developed RAM are compared as against the observations from conducting the practical risk assessment approach using traditional risk matrix. To do so, the involved experts in the study were asked to assign any numbers within the range of $[1,5]$ (as proposed in the traditional risk matrix (Baybutt, 2018)) to the probability and severity of the identified risks. Table 9 illustrates the responses of the experts, the aggregated magnitude obtained from all the experts' analyses, and the location of the analysed risks on the common risk matrix. Figure 3 also illustrates the obtained rankings of the analysed risks from the application of two methods.

Table 9. The results of practical risk assessment approach

\begin{tabular}{|c|c|c|c|c|c|c|c|c|}
\hline \multirow{2}{*}{ Risks } & \multicolumn{3}{|c|}{$\mathrm{P}$} & \multicolumn{3}{|c|}{$S$} & \multirow{2}{*}{$\begin{array}{l}\text { Aggregated } \\
\text { magnitude }\end{array}$} & \multirow{2}{*}{$\begin{array}{c}\text { Criticality } \\
\text { level }\end{array}$} \\
\hline & E1 & E2 & E3 & E1 & E2 & E3 & & \\
\hline S1 & 2 & 2 & 3 & 4 & 5 & 4 & 10.11 & High \\
\hline S2 & 1 & 1 & 2 & 4 & 5 & 4 & 5.77 & Medium \\
\hline S3 & 3 & 5 & 5 & 3 & 3 & 3 & 13 & High \\
\hline S4 & 2 & 3 & 3 & 4 & 5 & 5 & 12.44 & High \\
\hline S5 & 3 & 4 & 3 & 2 & 4 & 3 & 10 & Medium \\
\hline S6 & 4 & 4 & 4 & 2 & 3 & 3 & 10.66 & High \\
\hline S7 & 2 & 2 & 1 & 2 & 2 & 2 & 3.33 & Low \\
\hline S8 & 2 & 3 & 1 & 2 & 2 & 2 & 4 & Low \\
\hline S9 & 2 & 2 & 2 & 2 & 2 & 2 & 4 & Low \\
\hline S10 & 2 & 2 & 2 & 2 & 2 & 2 & 4 & Low \\
\hline S11 & 2 & 2 & 2 & 3 & 3 & 3 & 6 & Medium \\
\hline
\end{tabular}

\section{Discussion}

Due to the fact that the traditional-based risk matrix approach is tangled with major shortcomings as mentioned previously, the current study develops RAM for overcoming the afore-mentioned problems. To do so, FBWM and IVFTOPSIS with the aim of obtaining a precise final ranking of the risks are coupled with each other. To show the practicability of the developed RAM, the case of workers involved in maintaining lifts was taken into consideration.

As regards the calculation of the importance of the two risk parameters in the area of construction safety risk assessment using FBWM, severity was believed to be more precarious than probability to endanger the lives of the involved workers (see Table 4). On the other hand, it was shown that trapping (S3), fall from height (S4), back injury (S6), and electrocution (S1) made up the bulk of whole weights, and as such, the concerned safety experts need to give special consideration to control the mentioned risks at the next stage (see Table 7). It is also found out that struck by falling objects (S5), fire (S2), eye injury (S11), tension neck syndrome (S8), and tendinitis (S7) ranked from the fifth to the ninth place, respectively. By contrast, dermatitis (S9) and scald (S10) were perceived to be the
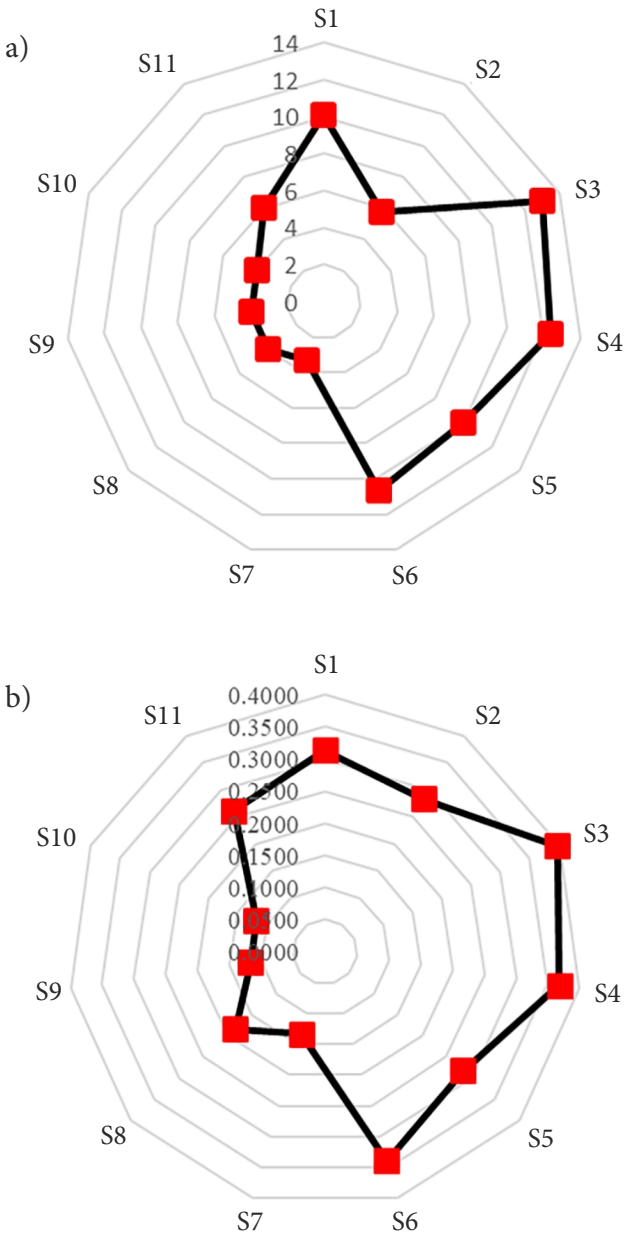

Figure 3. Analysis of the risks ranking with regard to final weights obtained from: (a) traditional risk matrix approach, (b) RAM approach

least critical risks, which require less attention to be paid upon than the others. The application of the developed model to the chosen case study shows clearly the ranking of the identified risks in order of criticality, providing the concerned safety experts with the hindsight to give priority for the mitigation of those ranked at the top of the list. For instance, in the chosen case study, the priority for mitigation needs to be given to trapping (S3), followed by fall from height (S4), back injury (S6), electrocution (S1), struck by falling objects (S5), fire (S2), eye injury (S11), tension neck syndrome (S8), tendinitis (S7), dermatitis (S9) and scald (S10).

Based on the comparisons made between the results produced by the application of RAM and conventional approach (see Figure 3), it can clearly be seen that the traditional risk matrix approach cannot bring about accurate rankings of the analysed risks. In other words, some of the risks with different levels of criticality are plotted in the same region, which in turn impairs the perceptions of the concerned safety professionals in adopting the fruitful mitigation actions in risk management. For instance, the risks S2 and S5 were regarded as medium using traditional risk matrix approach, although the criticalities regarding 
these risks were high using the developed RAM. This indicates that the developed RAM is stricter than the conventional risk matrix assessment towards the classifications of the analysed risks, culminating in more circumspect decisions. The obtained rankings and classifications from the application of the RAM to the chosen case study are in line with the studies conducted by Lee and Lim (2014), McCann (2013), Mohandes and Zhang (2019), Zarikas et al. (2013), in which S1, S3, S4, and S6 were concluded as the most critical risks that endangered the lives of workers involved in maintaining lifts. All in all, the fallacy of the practical risk-matrix-based approach is due to the following reasons:

1) Traditional risk matrix cannot differentiate the importance of the two essential risk parameters in assessing the respective risks related to the construction workers. That is to say, the severity and probability parameters are viewed with the same level of importance, which leads to distorting the obtainment of prudent analyses (e.g. a risk with the probability and severity values of 2 and 3 respectively has the same magnitude with a risk whose assigned probability and severity values are 3 and 2 respectively);

2) The ratings used in the traditional risk matrix cannot overcome the human's subjectivity in assessing the related risks to the construction workers, since the safety analysts should use only raw numbers;

3) Due to the limited range of the classifications used for analysing risks in the common risk matrix approach, the analysed risks could not be properly classified based on their criticalities. In other words, the respective safety analyst in assigning a value to the probability or severity of the risk has solely 5 ranges of classifications (i.e. very low, low, medium, high, very high (Baybutt, 2018)). Consequently, this limitation leads to the fact that some risks, which have different magnitude, are plotted in the same location on the final risk matrix. Ultimately, this fact culminates in impairing the proposal of suitable mitigation actions in the subsequent step.

\section{Conclusions}

In this study, a novel risk assessment model is developed to overcome the incompletion and uncertainty existing in the practical risk assessment approach using traditional risk matrix, including the overlooking the importance of the two essential risk parameters, inability to deal with the safety analysts' subjectivity, and curbed range of classifications. To this end, the FIS is integrated with two MCDM methods to systematically obtain a precise final ranking of the risks threatening the involved workers' lives. As the first part of the developed model, the FBWM is used to calculate the importance weights of the two risk parameters, which are used as the inputs to the proposed IVFTOPSIS. Through the proposed IVFTOPSIS, final rankings of the risks can be obtained. Finally, some rules along with a number of considerations are proposed for dealing with the analysed risks in the step of evaluation. With a view to showing the practicability of the integrated RAM, the case of workers involved in the maintenancerelated activities to the lifts was chosen. Based on the application of the developed assessment model to the chosen case study, it is observed that the developed RAM contributes to the body of construction safety risk assessment in five ways as follows:

1) The importance of the two essential risk parameters, i.e. probability and severity, can precisely be computed using fuzzy-reference-based comparisons;

2) There is no need for obtaining statistical data that are mostly associated with uncertainty and incompletion;

3) The identified risks can systematically be prioritized using the combination of interval-valued triangular fuzzy numbers and TOPSIS approach;

4) Providing the safety analysts with wider ranges of classifications for conducting risk assessment step related to the construction workers using 7-fuzzybased linguistic scales; and

5) Providing the concerned safety professionals with prudent evaluation strategies to control the analysed risks in order of criticality in the next step.

The developed model in the study can be applied to any project that gives the required hindsight to the safety experts to take the further prudent mitigation measurements, improving the involved workers' safety and health. As explained extensively, the developed model in the study leads to the provisions of more sparse and lucid ranking of the analysed risks, which is in striking contrast with the traditional-based-risk-matrix approach. The resultant diversified ranking improves the adoption of further mitigation measures, improving the workers' safety and health. The future research can be conducted towards the integration of the other MCDM methods with FIS, and accordingly the produced results can be compared as against the results of the assessment model developed in the current study. In addition to that, newly-developed FISs can be used as an extension to the TFNs used in the current study (such as Interval-valued fuzzy sets, Pythagorean fuzzy sets, Intuitionistic fuzzy sets, etc.), and subsequently the results could be compared to those if this study. Moreover, the future studies can focus on extending the dimensions of the traditional risk matrix. Moreover, software or mobile application to support huge amount of mathematical operations could be another stream for conducting the future research.

Finally, this study used three experts for the validation of the developed assessment model, due to unavailability or reluctance of more experts for involving in the research. Although the practicability of the developed model in the study was shown using a panel of three experts, it is needed to apply the developed model to different case studies to be ensured regarding its efficacy in identifying, assessing, and evaluating the risks using different sizes of panel members. 


\section{Acknowledgements}

The authors would like to immensely thank Mr. Chau for his support and patience towards conducting the current research.

\section{Conflict of interest}

The authors declare no conflict of interest.

\section{References}

Abdel-Basset, M., Saleh, M., Gamal, A., \& Smarandache, F. (2019). An approach of TOPSIS technique for developing supplier selection with group decision making under type-2 neutrosophic number. Applied Soft Computing, 77, 438-452. https://doi.org/10.1016/j.asoc.2019.01.035

Aminbakhsh, S., Gunduz, M., \& Sonmez, R. (2013). Safety risk assessment using analytic hierarchy process (AHP) during planning and budgeting of construction projects. Journal of Safety Research, 46, 99-105.

https://doi.org/10.1016/j.jsr.2013.05.003

Amiri, M., Ardeshir, A., \& Fazel Zarandi, M. H. (2017). Fuzzy probabilistic expert system for occupational hazard assessment in construction. Safety Science, 93, 16-28.

https://doi.org/10.1016/j.ssci.2016.11.008

Aneziris, O. N., Papazoglou, I. A., \& Kallianiotis, D. (2010). Occupational risk of tunneling construction. Safety Science, 48(8), 964-972. https://doi.org/10.1016/j.ssci.2009.11.003

Armaghani, D. J., Mohamad, E. T., Momeni, E., \& Narayanasamy, M. S. (2015). An adaptive neuro-fuzzy inference system for predicting unconfined compressive strength and Young's modulus: a study on Main Range granite. Bulletin of Engineering Geology and the Environment, 74(4), 1301-1319. https://doi.org/10.1007/s10064-014-0687-4

Ashtiani, B., Haghighirad, F., Makui, A., \& ali Montazer, G. (2009). Extension of fuzzy TOPSIS method based on interval-valued fuzzy sets. Applied Soft Computing, 9(2), 457-461. https://doi.org/10.1016/j.asoc.2008.05.005

Baybutt, P. (2018). Guidelines for designing risk matrices. Process Safety Progress, 37(1), 49-55.

https://doi.org/10.1002/prs.11905

Bejarbaneh, B. Y., Bejarbaneh, E. Y., Fahimifar, A., Armaghani, D. J., \& Majid, M. Z. A. (2018). Intelligent modelling of sandstone deformation behaviour using fuzzy logic and neural network systems. Bulletin of Engineering Geology and the Environment, 77(1), 345-361.

https://doi.org/10.1007/s10064-016-0983-2

Celik, E., Gul, M., Gumus, A. T., \& Guneri, A. F. (2012). A fuzzy TOPSIS approach based on trapezoidal numbers to material selection problem. Journal of Information Technology Applications \& Management, 19(3), 19-30.

Debnath, J., Biswas, A., Sivan, P., Sen, K. N., \& Sahu, S. (2016). Fuzzy inference model for assessing occupational risks in construction sites. International Journal of Industrial Ergonomics, 55, 114-128. https://doi.org/10.1016/j.ergon.2016.08.004

Djapan, M., Macuzic, I., Tadic, D., \& Baldissone, G. (2018). An innovative prognostic risk assessment tool for manufacturing sector based on the management of the human, organizational and technical/technological factors. Safety Science, 119, 280-291. https://doi.org/10.1016/j.ssci.2018.02.032
Faber, M. H., \& Stewart, M. G. (2003). Risk assessment for civil engineering facilities: critical overview and discussion. Reliability Engineering \& System Safety, 80(2), 173-184. https://doi.org/10.1016/S0951-8320(03)00027-9

Gunduz, M., Birgonul, M. T., \& Ozdemir, M. (2016). Fuzzy structural equation model to assess construction site safety performance. Journal of Construction Engineering and Management, 143(4), 4016112.

https://doi.org/10.1061/(ASCE)CO.1943-7862.0001259

Guo, S., \& Zhao, H. (2017). Fuzzy best-worst multi-criteria decision-making method and its applications. Knowledge-Based Systems, 121, 23-31.

https://doi.org/10.1016/j.knosys.2017.01.010

Hallowell, M. R. (2008). A formal model for construction safety and health risk management. Oregon State University.

Hatami-Marbini, A., Tavana, M., Moradi, M., \& Kangi, F. (2013). A fuzzy group Electre method for safety and health assessment in hazardous waste recycling facilities. Safety Science, 51(1), 414-426. https://doi.org/10.1016/j.ssci.2012.08.015

Hinze, J., Devenport, J. N., \& Giang, G. (2006). Analysis of construction worker injuries that do not result in lost time. Journal of Construction Engineering and Management, 132(3), 321-326. https://doi.org/10.1061/(ASCE)0733-9364(2006)132:3(321)

Hwang, C. L., \& Yoon, K. (1981). Multiple attribute decision making: Methods and applications. New York: Springer-Verlag. https://doi.org/10.1007/978-3-642-48318-9

International Labour Organization. (2014, August 24-27). Safety and health at work: A vision for sustainable prevention. In XX World Congress on Safety and Health at Work 2014: Global Forum for Prevention, Frankfurt, Germany.

Lee, C. Y., \& Lim, C. H. (2014, December 9-12). Risk analysis and rescue operation for machine roomless lift: A case study. In 2014 IEEE International Conference on Industrial Engineering and Engineering Management, Bandar Sunway, Malaysia. https://doi.org/10.1109/IEEM.2014.7058841

Li, J., Bao, C., \& Wu, D. (2018). How to design rating schemes of risk matrices: A sequential updating approach. Risk Analysis, 38(1), 99-117. https://doi.org/10.1111/risa.12810

Liou, J. J. H., Yen, L., \& Tzeng, G.-H. (2008). Building an effective safety management system for airlines. Journal of Air Transport Management, 14(1), 20-26.

https://doi.org/10.1016/j.jairtraman.2007.10.002

Lu, S. T., Lin, C. W., \& Ko, P. H. (2008, September 5-7). Application of Analytic Network Process (ANP) in assessing construction risk of urban bridge project. In Second International Conference on Innovative Computing, Information and Control, ICICIC 2007, Kumamoto, Japan. https://doi.org/10.1109/ICICIC.2007.172

McCann, M. (2013). Deaths and injuries involving elevators and escalators. Silver Spring, MD: The Center for Construction Research and Training.

Mikaeil, R., Ozcelik, Y., Yousefi, R., Ataei, M., \& Hosseini, S. M. (2013). Ranking the sawability of ornamental stone using Fuzzy Delphi and multi-criteria decision-making techniques. International Journal of Rock Mechanics and Mining Sciences, 58, 118-126. https://doi.org/10.1016/j.ijrmms.2012.09.002

Mitropoulos, P., \& Namboodiri, M. (2010). New method for measuring the safety risk of construction activities: Task demand assessment. Journal of Construction Engineering and Management, 137(1), 30-38.

https://doi.org/10.1061/(ASCE)CO.1943-7862.0000246 
Mohandes, S. R., \& Zhang, X. (2019). Towards the development of a comprehensive hybrid fuzzy-based occupational risk assessment model for construction workers. Safety Science, 115, 294-309. https://doi.org/10.1016/j.ssci.2019.02.018

Mohandes, S. R., Zhang, X., \& Mahdiyar, A. (2019). A comprehensive review on the application of artificial neural networks in building energy analysis. Neurocomputing, 340, 55-75. https://doi.org/10.1016/j.neucom.2019.02.040

Mondal, S. P. (2016). Differential equation with interval valued fuzzy number and its applications. International Journal of System Assurance Engineering and Management, 7(3), 370386. https://doi.org/10.1007/s13198-016-0474-7

Nilsen, T., \& Aven, T. (2003). Models and model uncertainty in the context of risk analysis. Reliability Engineering \& System Safety, 79(3), 309-317.

https://doi.org/10.1016/S0951-8320(02)00239-9

Papazoglou, I. A., Aneziris, O. N., Bellamy, L. J., Ale, B. J. M., \& Oh, J. (2017). Quantitative occupational risk model: Single hazard. Reliability Engineering and System Safety, 160, 162173. https://doi.org/10.1016/j.ress.2016.12.010

Pinto, A. (2014). QRAM a qualitative occupational safety risk assessment model for the construction industry that incorporate uncertainties by the use of fuzzy sets. Safety Science, 63, 57-76. https://doi.org/10.1016/j.ssci.2013.10.019

Pinto, A., Nunes, I. L., \& Ribeiro, R. A. (2011). Occupational risk assessment in construction industry - overview and reflection. Safety Science, 49(5), 616-624.

https://doi.org/10.1016/j.ssci.2011.01.003

Rausand, M. (2013). Risk assessment: Theory, methods, and applications. John Wiley \& Sons.

https://doi.org/10.1002/9781118281116.ch8

Ren, Z., Xu, Z., \& Wang, H. (2017). Dual hesitant fuzzy VIKOR method for multi-criteria group decision making based on fuzzy measure and new comparison method. Information Sciences, 388-389, 1-16.

https://doi.org/10.1016/j.ins.2017.01.024

Sun, C.-C. (2010). A performance evaluation model by integrating fuzzy AHP and fuzzy TOPSIS methods. Expert Systems with Applications, 37(12), 7745-7754. https://doi.org/10.1016/j.eswa.2010.04.066

Tam, C. M., Zeng, S. X.,\& Deng, Z. M. (2004). Identifying elements of poor construction safety management in China. Safety Science, 42(7), 569-586. https://doi.org/10.1016/j.ssci.2003.09.001

Tzeng, G.-H., \& Huang, J.-J. (2011). Multiple attribute decision making: Methods and applications. CRC press. https://doi.org/10.1201/b11032

Wang, Y. M., Liu, J., \& Elhag, T. M. S. (2008). An integrated AHP-DEA methodology for bridge risk assessment. Computers and Industrial Engineering, 54(3), 513-525. https://doi.org/10.1016/j.cie.2007.09.002

Yazdi, M. (2018). Risk assessment based on novel intuitionistic fuzzy-hybrid-modified TOPSIS approach. Safety Science, 110, 438-448. https://doi.org/10.1016/j.ssci.2018.03.005

Zadeh, L. A. (1965). Fuzzy sets. Information and Control, 8(3), 338-353. https://doi.org/10.1016/S0019-9958(65)90241-X

Zarikas, V., Loupis, M., Papanikolaou, N., \& Kyritsi, C. (2013). Statistical survey of elevator accidents in Greece. Safety Science, 59, 93-103. https://doi.org/10.1016/j.ssci.2013.05.001

Zayed, T., Amer, M., \& Pan, J. (2008). Assessing risk and uncertainty inherent in Chinese highway projects using AHP.
International Journal of Project Management, 26(4), 408-419. https://doi.org/10.1016/j.ijproman.2007.05.012

Zhang, H., Xie, J., Lu, W., Zhang, Z., \& Fu, X. (2019). Novel ranking method for intuitionistic fuzzy values based on information fusion. Computers and Industrial Engineering, 133, 139-152. https://doi.org/10.1016/j.cie.2019.05.006

Zhao, D., McCoy, A., Kleiner, B., \& Feng, Y. (2016). Integrating safety culture into OSH risk mitigation: a pilot study on the electrical safety. Journal of Civil Engineering and Management, 22(6), 800-807. https://doi.org/10.3846/13923730.2014.914099

\section{APPENDIX}

\section{A. Designed questionnaire for FBWM}

Considering the calculation of the importance weights of the two risk parameters (i.e. probability and severity), select the MORE IMPORTANT parameter compared to the other one in the area of construction safety risk assessment (fill in the row highlighted in orange). Afterwards, you need to define the extent of the importance of the chosen MORE IMPORTANT parameter as against the other one using fuzzy variables (fill in the boxes highlighted in blue).

\begin{tabular}{|c|c|c|}
\hline The MORE IMPORTANT parameter & Probability & Severity \\
\hline Severity & M & E \\
\hline
\end{tabular}

\section{B. Calculating the importance weights of probability and severity using FBWM}

Regarding one of the experts' responses as illustrated in the previous Appendix, the computational steps required for calculating the importance weights of two risk parameters using FBWM are explained hereinafter.

Step 1. $B=$ Severity $(S)$, and $W=$ Probability $(P)$;

Step 2. $\tilde{a}_{B W}=M$;

Step 3. Based on the first and second step, we can achieve the following constrained optimization model:

$\min \widetilde{\xi^{*}}$

$$
\text { s.t. }\left\{\begin{array}{l}
\mid\left(\frac{\left.l_{S}^{w}, m_{S}^{w}, u_{S}^{w}\right)}{\left(l_{P}^{w}, m_{P}^{w}, u_{P}^{w}\right)}-\left(l_{S P}, m_{S P}, u_{S P}\right) \mid \leq\left(k^{*}, k^{*}, k^{*}\right)\right. \\
\sum_{j=1}^{n} R\left(\tilde{w}_{j}\right)=1 \\
l_{j}^{w} \leq m_{j}^{w} \leq u_{j}^{w} \\
l_{j}^{w} \geq 0 \\
j=1,2
\end{array} .\right.
$$

Replacing $\tilde{w}_{B}=\left(l_{S}^{w}, m_{S}^{w}, u_{S}^{w}\right)$ and $\tilde{w}_{W}=\left(l_{P}^{w}, m_{P}^{w}, u_{P}^{w}\right)$ by $\tilde{w}_{1}=\left(l_{1}, m_{1}, u_{1}\right)$ and $\tilde{w}_{2}=\left(l_{2}, m_{2}, u_{2}\right)$ respectively, we can obtain the below-mentioned model demonstrated by concrete numbers. 
$\min k^{*}$

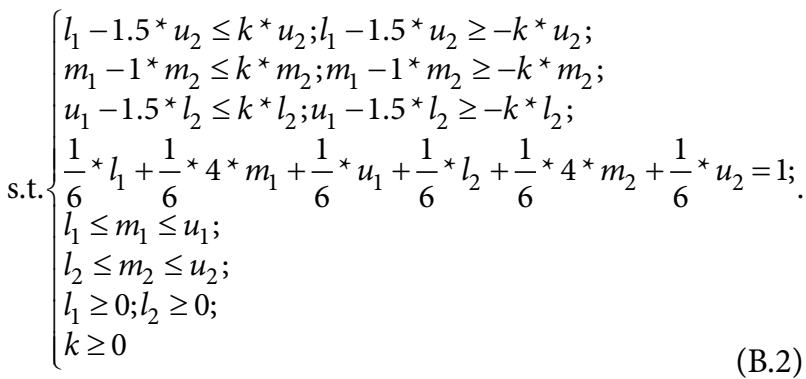

Considering the above-mentioned model, we have $\tilde{w}_{1}^{*}$ and $\tilde{w}_{2}^{*}$ as $(0.5854,0.6626,0.7489)$ and $(0.2995,0.3313,0.3903)$, respectively. Afterwards, the crisp weights of the importance of probability and severity using GMIR can be obtained as follows; $\tilde{w}_{1}^{*}=0.6641$ and $\tilde{w}_{2}^{*}=0.3358$.

\section{The sample questionnaire of IVFTOPSIS (evaluating the probability of risk factors)}

Considering the obtainment of the risks ranking, evaluate the listed risk factors with respect to the two risk parameters (i.e. probability and severity). In other words, you are asked to define the extent of occurrence (i.e. probability) and the extent of the criticality (i.e. severity) of all the listed risk factors using linguistic variables (fill the boxes highlighted in blue).

\begin{tabular}{|l|c|c|}
\hline \multicolumn{1}{|c|}{ Factors } & Probability & Severity \\
\hline Electrocution & VLP & VHP \\
\hline Fire & ELP & VHP \\
\hline Trapping & MHP & MHP \\
\hline Fall from height & MLP & VHP \\
\hline Struck & MHP & MLP \\
\hline Back injury & VHP & MLP \\
\hline Tendinitis & MLP & VLP \\
\hline Neck injury & MHP & VLP \\
\hline Dermatitis & VLP & VLP \\
\hline Scald & VLP & VLP \\
\hline Eye injury & MLP & MP \\
\hline
\end{tabular}

